\title{
THE CANONICAL SCHOENFLIES THEOREM
}

\author{
DAVID B. GAULD
}

ABstract. M. Brown has shown that if $f: S^{n-1} \times I^{\prime} \rightarrow S^{n}$, where $I^{\prime}=[-1,1]$, is an embedding, then the closure of either complementary domain of $f\left(S^{n-1} \times 0\right)$ is homeomorphic to $B^{n}$; in fact there is an embedding $g: B^{n} \rightarrow S^{n}$ satisfying $g\left|S^{n-1}=f\right| S^{n-1} \times 0$. This paper shows that the choice of embedding $g$ can be made to be "canonical," i.e. if $f^{\prime}$ is an embedding near $f$ in the compact-open sense, then the embedding $g^{\prime}$ corresponding to $f^{\prime}$ is near $g$.

1. Introduction. Brown [1] has proven the following result, the generalised Schoenflies theorem.

Let $f: S^{n-1} \times I^{\prime} \rightarrow S^{n}$ be an embedding, where $I^{\prime}=[-1,1]$. Then the closure of either complementary domain of $f\left(S^{n-1} \times 0\right)$ is homeomorphic to $B^{n}$.

Thus if we let $\varepsilon\left[S^{n-1} \times I^{\prime}, S^{n}\right]$ denote the collection of embeddings of $S^{n-1} \times I^{\prime}$ in $S^{n}$ and $\varepsilon\left[B^{n}, S^{n}\right]$ the collection of embeddings of $B^{n}$ in $S^{n}$, then Brown's result gives a function

$$
\Phi: \varepsilon\left[S^{n-1} \times I^{\prime}, S^{n}\right] \rightarrow \varepsilon\left[B^{n}, S^{n}\right]
$$

such that for all $f \in \mathcal{E}\left[S^{n-1} \times I^{\prime}, S^{n}\right]$ and all $x \in S^{n-1}$, we have $\Phi(f)(x)$ $=f(x, 0)$, and that for all $f \in \mathcal{E}\left[S^{n-1} \times I^{\prime}, S^{n}\right], f\left(S^{n-1} \times 1\right) \subset \Phi(f)\left(B^{n}\right)$.

Now $\mathcal{E}\left[S^{n-1} \times I^{\prime}, S^{n}\right]$ and $\mathcal{E}\left[B^{n}, S^{n}\right]$ can be topologised by the compact-open topology. Thus we might ask whether such a function $\Phi$ can be chosen to be continuous. Huebsch and Morse [2] have shown this to be the case. Here the same result is proven by showing that all choices in Brown's proof can be made continuously.

Brown's proof is essentially carried out in two steps: it is shown that for all $f \in \mathcal{E}\left[S^{n-1} \times I^{\prime}, S^{n}\right]$, there exists $g \in \mathcal{E}\left[B^{n}, S^{n}\right]$ such that $A_{f} \subset g\left(\right.$ Int $\left.B^{n}\right)$ and $B_{f} \cap g\left(B^{n}\right)=\varnothing, A_{f}$ and $B_{f}$ being the closed complementary domains of $f\left(S^{n-1} \times I^{\prime}\right)$ defined below. The second stage shows that, given such a pair $(f, g)$, one can construct the required embedding $\Phi(f)$.

The proof of continuity at the second stage is effectively included in the proof of Theorem 1 of Kister [3], so we will not verify it here. However, we make some remarks which indicate how Kister's proof can be adapted to our case.

Received by the editors January 27, 1970.

AMS 1969 subject classifications. Primary 5478, 5428, 5570, 5705.

Key words and phrases. Embedding spheres in spheres, locally-flat embeddings, Schoenflies theorem. 
(i) Although Kister's expansion of his embedding $g$ by the isotopy $F_{t}$ moves every point except $g(0)=0$, the isotopies $\alpha_{t}$ and $\beta_{t}$ could be chosen so that $F_{t} \nmid r B^{n}=g \nmid r B^{n}$ for some canonically chosen positive number $r$ are satisfying: $\exists s \in(0, \infty)$ such that $g\left(r B^{n}\right) \subset s B^{n} \subset g\left(R^{n}\right)$.

(ii) The expansion $F_{t}$ is radial in the sense that if $g^{\prime}$ is another embedding, with $F_{t}$ the corresponding isotopy, and $\exists$ homeomorphism $h: R^{n} \rightarrow R^{n}$ with $h g=g^{\prime}$ and $h(r x)=r h(x) \forall x \in R^{n}, \forall r \in R_{+}$, then $h F_{t}$ $=F_{t}^{\prime}$.

(iii) A consequence of (i) is that to construct $F_{t}$, we need only the radial structure of $R^{n}-g\left(r B^{n}\right) \subset R^{n}-$ Int $q B^{n}$ for some positive number $q$. Now this latter space, $R^{n}-$ Int $q B^{n}$ is a half-open annulus $S^{n-1} \times[0,1)$, there being a natural homeomorphism which preserves the radial structure. By (ii), only the radial structure of this halfopen annulus is used.

(iv) We may therefore restate Kister's result in the following way: Given a manifold $M^{n}$ and two embeddings

$$
f: S^{n-1} \times[-1,1] \rightarrow M, \quad g: S^{n-1} \times[0,1) \rightarrow M,
$$

satisfying

(a) $f\left(S^{n-1} \times 1\right) \subset g\left(S^{n-1} \times(0,1)\right)$,

(b) $g\left(S^{n-1} \times 0\right) \subset f\left(S^{n-1} \times(0,1)\right)$,

we can expand the embedding $f \mid S^{n-1} \times[0,1)$ by an isotopy $F_{t}$ so that $F_{t}\left|S^{n-1} \times 0=f\right| S^{n-1} \times 0$, and so that $g\left(S^{n-1} \times[0,1)\right) \subset F_{1}\left(S^{n-1} \times[0,1)\right)$. Think of our $f$ as a restriction of Kister's $g$, our $g$ providing the radial structure. Moreover, this isotopy $F_{t}$ depends canonically on $f$ and $g$ and depends only on the radial structure given to $g\left(S^{n-1} \times[0,1)\right)$ by $g$; i.e. if $h: S^{n-1} \rightarrow S^{n-1}$ is any homeomorphism, then the pair of embeddings $f$ and $g(h \times 1)$ give rise to the same isotopy $F_{t}$.

By noting that $S^{n-1} \times[0,1)$ is homeomorphic to $B^{n}-\{0\}$, we see now that Kister's proof gives us a map

$$
\begin{array}{r}
\Psi:\left\{(f, g) \in \varepsilon\left[S^{n-1} \times I^{\prime}, S^{n}\right] \times \varepsilon\left[B^{n}, S^{n}\right] / A_{f} \subset g\left(\operatorname{Int} B^{n}\right)\right. \\
\text { and } \left.B_{f} \cap g\left(B^{n}\right)=\varnothing\right\} \rightarrow \varepsilon\left[B^{n}, S^{n}\right]
\end{array}
$$

such that $\Psi(f, g)(x)=f(x, 0)$ for all $x \in S^{n-1}=\partial B^{n}$. Further, $\Psi\left(f, g_{1}\right)$ $=\Psi\left(f, g_{2}\right)$ whenever $g_{1}$ and $g_{2}$ are radially equivalent, where

DEFinition. Two embeddings $g_{1}, g_{2}: B^{n} \rightarrow S^{n}$ are radially equivalent iff $\exists$ a homeomorphism $h: B^{n} \rightarrow B^{n}$ with $h(t x)=t h(x) \quad \forall x \in B^{n}$ and $\forall t \in I$ and $g_{1}=g_{2} h$. In such a case we write $g_{1} \sim g_{2}$. $\sim$ is, of course, an equivalence relation.

We will verify continuity at the first stage, constructing twelve maps, the last of which is

$$
\nu: \varepsilon\left[S^{n-1} \times I^{\prime}, S^{n}\right] \rightarrow \varepsilon\left[B^{n}, S^{n}\right] / \sim
$$


such that for all $f \in \varepsilon\left[S^{n-1} \times I^{\prime}, S^{n}\right]$ and $g \in \nu(f), A_{f} \subset g\left(\right.$ Int $\left.B^{n}\right)$ and $B_{f} \cap g\left(B^{n}\right)=\varnothing$.

If, then, we define $\Phi(f)=\Psi(f, g)$ where $g \in \nu(f)$, we have the following result:

Canonical Schoenflies Theorem. There exists a map

$$
\Phi: \varepsilon\left[S^{n-1} \times I^{\prime}, S^{n}\right] \rightarrow \varepsilon\left[B^{n}, S^{n}\right]
$$

such that for all $x \in S^{n-1}=\partial B^{n}, \Phi(f)(x)=f(x, 0)$.

We require some notation. If $X$ and $Y$ are two topological spaces, then $\varepsilon[X, Y], \mathfrak{T}[X, Y]$ and $\mathscr{H}(X)$ denote respectively the spaces of embeddings of $X$ in $Y$, of maps from $X$ to $Y$ and of homeomorphisms of $X$, all with the compact-open topologies.

$\rho: S^{n-1} \times I^{\prime} \rightarrow S^{n}$ is defined by $\rho\left(x_{0}, \cdots, x_{n-1}, t\right)=\left(x_{0} \sqrt{ }\left(1-t^{2}\right), \cdots\right.$, $\left.x_{n-1} \sqrt{ }\left(1-t^{2}\right), t\right) . N=$ north pole of $S^{n}=(0, \cdots, 0,1) ; E=$ east pole of $S^{n}=(1,0, \cdots, 0)$ (note that $\left.E \in S^{n-1}\right) ; N^{\prime}=$ north pole of $S^{n-1}$ $=(0, \cdots, 0,1)$. If $x, y \in R^{n}$, then $|x-y|$ is the usual distance between $x$ and $y$. If $x \in S^{n}$ and $r \in(0, \infty), D(x ; r)=\left\{y \in S^{n} /|x-y| \leqq r\right\}$. Let $V=\left\{x \in S^{n} / x=(\cos \theta, 0, \cdots, 0, \sin \theta)\right.$ for some $\left.\theta \in[2 \pi / 3,3 \pi / 4]\right\}$ and $U=\left\{y \in S^{n} / \exists x \in V\right.$ so that $\left.|x-y| \leqq 1 / 10\right\}$. Then $U$ is a neighbourhood of $V$ not containing $N$ and not meeting the equator. See Figure 1.

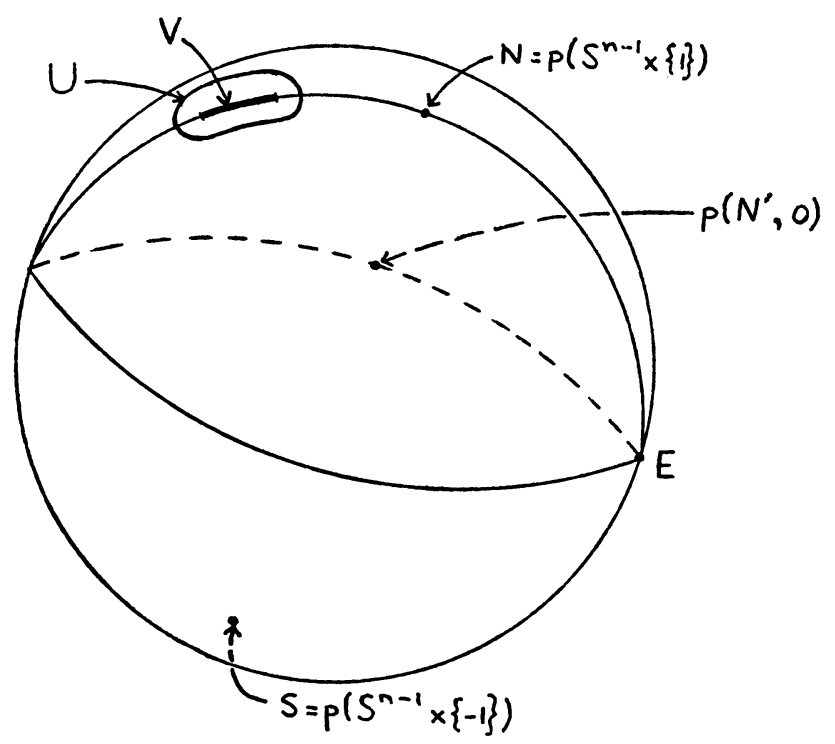

FIGURE 1 


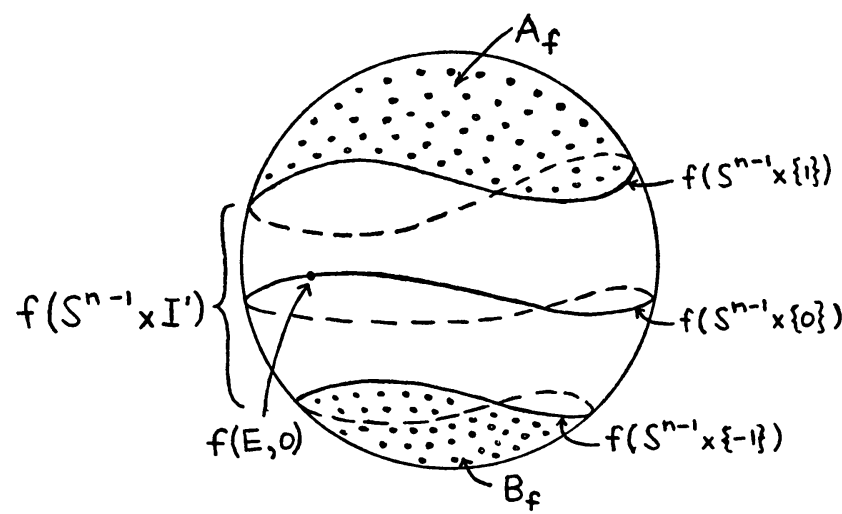

FIGURE 2. The Embedding $f$

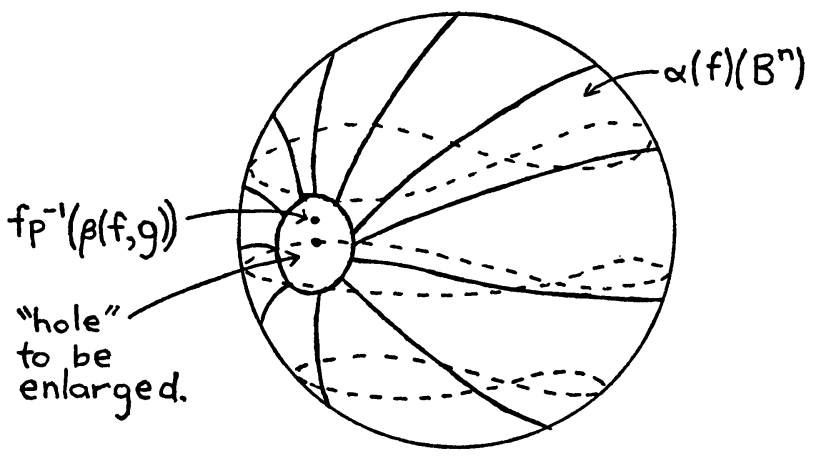

FIGURE 3

If $f \in \varepsilon\left[S^{n-1} \times I^{\prime}, S^{n}\right]$, let $A_{f}$ be that component of the closure of $S^{n}-f\left(S^{n-1} \times I^{\prime}\right)$ containing $f\left(S^{n-1} \times 1\right)$ and $B_{f}$ that component containing $f\left(S^{n-1} \times\{-1\}\right)$. See Figure 2. For all $x \in S^{n}, g_{x}: S^{n}-\{x\} \rightarrow R^{n}$ is a stereographic projection from $x$ sending $\left\{y \in S^{n} /|x-y|=\sqrt{ } 2\right\}$ to $S^{n-1}$. Now $g_{x}$ is not well defined, but we require $g_{x}$ only to impose a radial structure on $S^{n}-\{x\}$, and this is independent of the choice of $g_{x}$.

If $g \in \mathcal{E}\left[B^{n}, S^{n}\right] / \sim$, denote by $\mathcal{G}\left(B^{n}\right)$ and $\mathcal{G}\left(\operatorname{Int} B^{n}\right)$ the sets $g\left(B^{n}\right)$ and $g\left(\operatorname{Int} B^{n}\right)$ for $g \in g$. These sets are well defined.

The present paper is part of the author's doctoral dissertation written under the guidance of Professor Robion C. Kirby at the University of California, Los Angeles. I wish to express my gratitude to Professor Kirby for his advice and encouragement.

2. Definition of the maps. We firstly choose, for all $f \in \varepsilon\left[S^{n-1} \times I^{\prime}, S^{n}\right]$, a canonical class of embeddings $\alpha(f) \in$ 
$\mathcal{E}\left[B^{n}, S^{n}\right] / \sim$ such that $A_{f} \cup B_{f} \subset \alpha(f)\left(\right.$ Int $\left.B^{n}\right)$. Precisely, define the map

$$
\alpha: \varepsilon\left[S^{n-1} \times I^{\prime}, S^{n}\right] \rightarrow \varepsilon\left[B^{n}, S^{n}\right] / \sim
$$

by letting $\alpha(f)$ be the equivalence class of embeddings $B^{n} \rightarrow S^{n}$ defined by

$$
y \rightarrow g_{f(E, 0)}^{-1}(r y),
$$

where $r=2 \min \left\{r \in(0, \infty) / g_{f(E, 0)}\left(A_{f} \cup B_{f}\right) \subset r B^{n}\right\}$. See Figure 3 .

We must now enlarge the "hole" $S^{n}-\alpha(f)\left(B^{n}\right)$, which lies entirely in $f\left(S^{n-1} \times(-1,1)\right)$, to include $B_{f}$ also. This enlargement takes several steps and requires the space

$$
a=\left\{(f, g) \in \varepsilon\left[S^{n-1} \times I^{\prime}, S^{n}\right] \times \varepsilon\left[B^{n}, S^{n}\right] / g \in \alpha(f)\right\} .
$$

The first few steps will culminate in a map

$$
\mu: a \rightarrow \varepsilon\left[B^{n}, S^{n}\right],
$$

with $A_{f} C \mu(f, g)\left(\right.$ Int $\left.B^{n}\right)$ and $B_{f} \cap \mu(f, g)\left(B^{n}\right)=\varnothing . \mu$ will be constructed in such a way that if $\left(f, g_{1}\right),\left(f, g_{2}\right) \in Q$, then $\mu\left(f, g_{1}\right)$ and $\mu\left(f, g_{2}\right)$ are radially equivalent. The required map

$$
\nu: \varepsilon\left[S^{n-1} \times I^{\prime}, S^{n}\right] \rightarrow \varepsilon\left[B^{n}, S^{n}\right] / \sim
$$

may then be defined by letting $\nu(f)$ be the equivalence class of $\mu(f, g)$ for $g \in \alpha(f)$.

The first step is to enlarge the "hole" $S^{n}-g\left(B^{n}\right)$ to include $A_{f}$. At the end of the step, we will have a map $\epsilon(f, g)$ which is an embedding on all of $B^{n}$ except $g^{-1}\left(A_{f}\right), \epsilon(f, g)$ sending this latter set to the point $\delta(f, g)$.

Define $\beta: Q \rightarrow S^{n}$ by letting $\beta(f, g)$ be that point in the northern hemisphere of $S^{n}$ lying on the great circle between $E$ and $N$ (i.e. all coordinates except the first and last are zero, and the last is positive) whose distance from $E$ is

$$
\frac{1}{2} \sup \left\{r \in(0,2) \mid D(E ; r) \cap p f^{-1} g\left(B^{n}\right)=\varnothing\right\} .
$$

Note that $\beta(f, g) \neq N$, so $f p^{-1}(\beta(f, g))$ is a single point. Note also that $f p^{-1}(\beta(f, g)) \notin g\left(B^{n}\right)$.

Define $\gamma: Q \rightarrow \mathfrak{C C}\left(S^{n}\right)$ by letting $\gamma(f, g)$ be the homeomorphism which leaves the southern hemisphere fixed, takes $\beta(f, g)$ to $N$ and moves the natural cone structure on the northern hemisphere with $\beta(f, g)$ as vertex and the equator as base to that with $N$ as vertex. Note that $\boldsymbol{\gamma}(f, g)(N) \in V$. See Figure 4. 


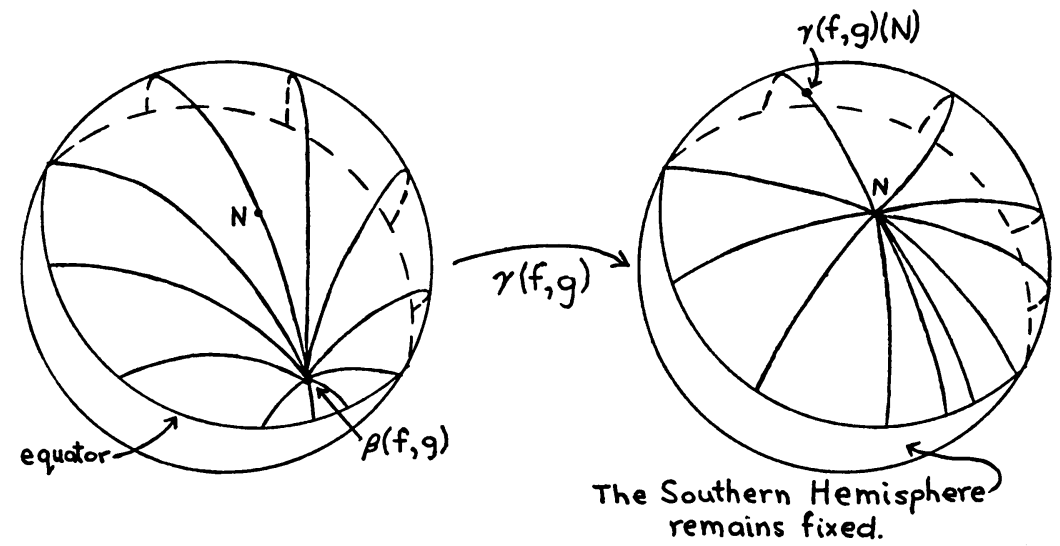

FIGURE 4

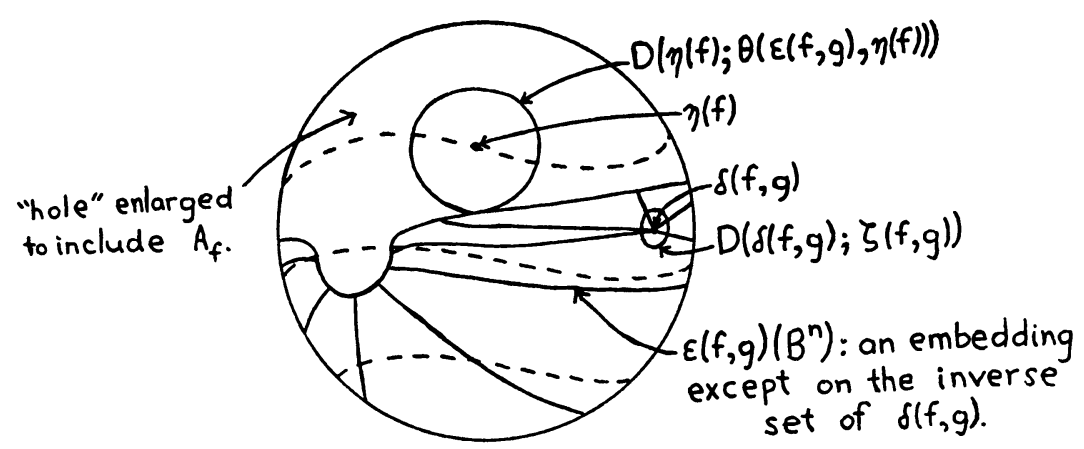

FIGURE 5

Define $\delta: a \rightarrow S^{n}$ by $\delta(f, g)=f p^{-1} \gamma(f, g)(N)$.

Define $\epsilon: Q \rightarrow \mathscr{T}\left[B^{n}, S^{n}\right]$ by

$$
\begin{aligned}
\epsilon(f, g)(x) & =\delta(f, g) & & \text { if } g(x) \in A_{f}, \\
& =f p^{-1} \gamma(f, g) p f^{-1} g(x) & & \text { if } g(x) \in f\left(S^{n-1} \times[0,1]\right), \\
& =g(x) & & \text { if } g(x) \in B_{f} \cup f\left(S^{n-1} \times[-1,0]\right) .
\end{aligned}
$$

See Figure 5.

The second step is to squeeze $\epsilon(f, g)\left(B^{n}\right)$ into a small neighbourhood $D(\delta(f, g) ; \zeta(f, g))$ of $\delta(f, g)$ in $S^{n}$, keeping a smaller neighbourhood fixed.

Define $\zeta: Q \rightarrow(0, \infty)$ by letting $\zeta(f, g)=\frac{1}{2} \max \left\{r \in(0, \infty) / D(\delta(f, g) ; r) \subset f p^{-1}(U) \cap \epsilon(f, g)\left(B^{n}\right)\right\}$.

Define $\eta: \varepsilon\left[S^{n-1} \times I^{\prime}, S^{n}\right] \rightarrow S^{n}$ by $\eta(f)=f\left(N^{\prime}, 1\right)$. 
Let $\theta:\left\{(h, x) \in \mathfrak{M}\left[B^{n}, S^{n}\right] \times S^{n} / x \notin h\left(B^{n}\right)\right\} \rightarrow(0, \infty)$ be given by $\theta(h, x)=\sup \left\{r \in(0, \infty) / D(x ; r) \cap h\left(B^{n}\right)=\varnothing\right\}$.

$\kappa:\left\{(x, y, r, s, t) \in S^{n} \times S^{n} \times(0, \infty) \times(0, \infty) \times(0, \infty) / r<s\right.$ and $D(x ; s) \cap D(y ; t)=\varnothing\} \rightarrow \mathcal{C}\left(S^{n}\right)$ is the following: $\kappa(x, y, r, s, t)$ fixes $D(x ; r) \cup\{y\}$, moves the collar $S^{n}$-Int $D(x ; r)-$ Int $D(y ; t)$ onto $D(x ; s)$-Int $D(x ; r)$ and expands $D(y ; t)$ to $S^{n}-\operatorname{Int} D(x ; s)$. This homeomorphism is defined as follows: let $z \in S^{n}$. If $z \in D(x ; r) \cup\{y\}$, set $\kappa(x, y, r, s, t)(z)=z$. If $z \notin\{x, y\}$, then $g_{x}(y)$ and $g_{x}(z)$ determine a ray in $R^{n}$. Let $u, v$ and $w$ denote the points where this ray meets $g_{x} \partial D(y ; t), g_{x} \partial D(x ; s)$ and $g_{x} \partial D(x ; r)$ respectively. Then let $\kappa(x, y, r, s, t)(z)$ be

$$
g_{x}^{-1}\left(\frac{\left|g_{x}(z)-u\right|}{\left|u-g_{x}(y)\right|} g_{x}(y)+\frac{\left|g_{x}(z)-g_{x}(y)\right|}{\left|u-g_{x}(y)\right|} v\right)
$$

if $z \in D(y ; t)$, and

$$
g_{x}{ }^{-1}\left(\frac{\left|g_{x}(z)-w\right|}{|u-w|} v+\frac{\left|g_{x}(z)-u\right|}{|u-w|} w\right)
$$

if $z \in S^{n}-$ Int $D(x ; r)-\operatorname{Int} D(y ; t)$.

Now note that

$$
D(\delta(f, g) ; \zeta(f, g)) \subset \epsilon(f, g)\left(\operatorname{Int} B^{n}\right)
$$

and

$$
D(\eta(f) ; \theta(\epsilon(f, g), \eta(f))) \cap \epsilon(f, g)\left(\text { Int } B^{n}\right)=\varnothing,
$$

so $D(\delta(f, g) ; \zeta(f, g)) \cap D(\eta(f) ; \theta(\epsilon(f, g), \eta(f)))=\varnothing$. Hence

$$
\left(\delta(f, g), \eta(f), \frac{1}{2} \zeta(f, g), \zeta(f, g), \theta(\epsilon(f, g), \eta(f))\right)
$$

is in the domain of $\kappa$. Thus we may define $\lambda: a \rightarrow \mathfrak{H C}\left(S^{n}\right)$ by

$$
\lambda(f, g)=\kappa\left(\delta(f, g), \eta(f), \frac{1}{2} \zeta(f, g), \zeta(f, g), \theta(\epsilon(f, g), \eta(f))\right) .
$$

Note that $\lambda(f, g) \mid D\left(\delta(f, g) ; \frac{1}{2} \zeta(f, g)\right)=1$ and that

$$
\lambda(f, g)\left(S^{n}-D(\eta(f) ; \theta(\epsilon(f, g), \eta(f)))\right) \subset D(\delta(f, g) ; \zeta(f, g)),
$$

so that $\lambda(f, g)$ does the required squeezing at the second step. See Figure 6.

Now define the map $\mu: Q \rightarrow \varepsilon\left[B^{n}, S^{n}\right]$ as follows. See Figure 7 .

$$
\begin{aligned}
\mu(f, g)(x) & =g(x) & & \text { if } g(x) \in A_{f}, \\
& =f p^{-1} \gamma(f, g)^{-1} p f^{-1} \lambda(f, g) \epsilon(f, g)(x) & & \text { otherwise. }
\end{aligned}
$$




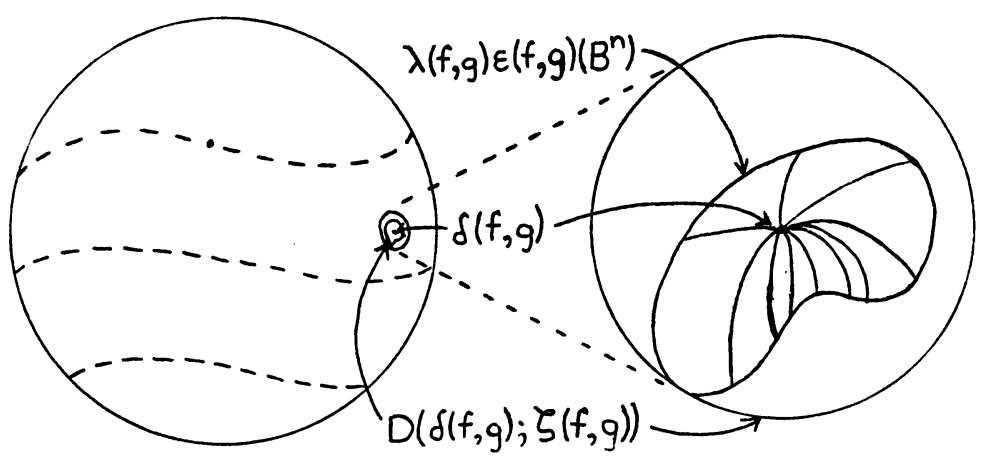

FIGURE 6

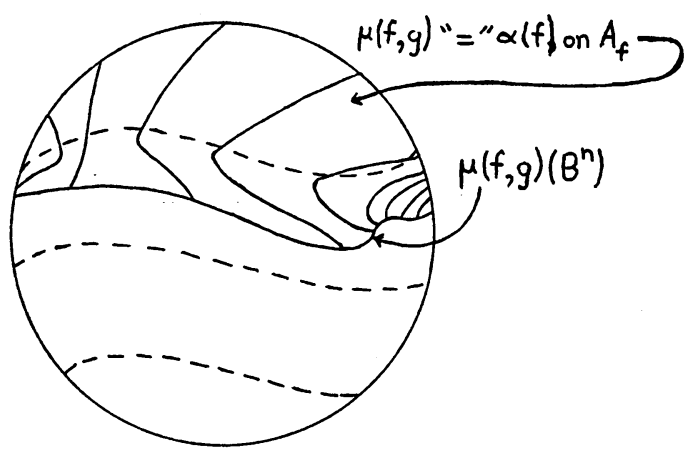

Figure 7

Note that if

$$
g(x) \in f\left(S^{n-1} \times[0,1]\right) \cap f p^{-1} \gamma(f, g)^{-1} p f^{-1}\left(D\left(\delta(f, g) ; \frac{1}{2} \zeta(f, g)\right)\right),
$$

which, together with $A_{f}$, forms a neighbourhood of $A_{f}$, then

$$
\mu(f, g)(x)=f p^{-1} \gamma(f, g)^{-1} p f^{-1} \lambda(f, g) f p^{-1} \gamma(f, g) p f^{-1} g(x)=g(x),
$$

so that $\mu(f, g)(x)=g(x)$ on this neighbourhood of $A_{f}$. Note also that $\mu(f, g)\left(B^{n}\right) \cap B_{f}=\varnothing$, so that if $\mu(f, g)$ is an embedding and $\mu$ is continuous, then $\mu$ satisfies all of the conditions required of it.

3. Well-definition and continuity of the maps. We firstly check the well-definition. That of $\alpha, \beta, \gamma, \delta, \eta, \theta, \kappa$ and $\lambda$ is clear. If $g(x) \in f\left(S^{n-1} \times[0,1]\right)$, then $\gamma(f, g) p f^{-1} g(x)$ lies in the northern hemisphere minus $N$, so $\epsilon(f, g)(x)$ is defined on this part of the domain. If $g(x) \in f\left(S^{n-1} \times 0\right)$, then $p f^{-1} g(x)$ lies on the equator which is fixed by $\gamma(f, g)$. Thus $f p^{-1} \gamma(f, g) p f^{-1} g(x)=g(x)$ here. If $g(x) \in f\left(S^{n-1} \times 1\right)$, then 
$p f^{-1} g(x)=N$, so that $f p^{-1} \gamma(f, g) p f^{-1} g(x)=\delta(f, g)$ in this case. Thus $\epsilon(f, g)$ is a well-defined function, and it is continuous since $f$ and $p \mid S^{n-1}$ $\times(-1,1)$ are embeddings. Thus $\epsilon$ is well-defined.

$U$ was chosen so that $\gamma(f, g)(N) \in$ Int $U$, so $\delta(f, g) \in f p^{-1}$ (Int $\left.U\right)$. Clearly $\delta(f, g) \in$ Int $\epsilon(f, g)\left(B^{n}\right)=\epsilon(f, g)$ (Int $\left.B^{n}\right)$. Thus

$$
\left\{r \in(0, \infty) / D(\delta(f, g) ; r) \subset f p^{-1}(U) \cap \epsilon(f, g)\left(B^{n}\right)\right\}
$$

is nonempty, so $\zeta$ is well-defined.

If $g(x) \in B_{f} \cup f\left(S^{n-1} \times[-1,1)\right)$, then

$$
\lambda(f, g) \epsilon(f, g)(x) \in D(\delta(f, g) ; \zeta(f, g)) \subset f\left(S^{n-1} \times[0,1)\right),
$$

but $\lambda(f, g) \epsilon(f, g)(x) \neq \delta(f, g)$. Hence $\gamma(f, g)^{-1} p f^{-1} \lambda(f, g) \epsilon(f, g)(x)$ lies in the northern hemisphere but is not $N$, so that $f p^{-1} \gamma(f, g)^{-1} p f^{-1}$ $\cdot \lambda(f, g) \epsilon(f, g)(x)$ is well-defined. As has already been noted, $\mu(f, g)$ agrees with $g$ on a neighbourhood of $g^{-1}\left(A_{f}\right)$. Thus $\mu(f, g)$ is continuous. $\mu(f, g)$ is clearly injective on each of the two parts of its domain, and the images of these parts do not overlap since

$$
A_{f} \cap f\left(S^{n-1} \times(-1,1)\right)=\varnothing .
$$

Thus $\mu(f, g)$ is injective and hence an embedding. Thus $\mu$ is welldefined.

One can readily check that, with the exception of $\epsilon$, none of the maps $\beta, \cdots, \lambda$ depends on $g$, i.e. the images of $\left(f, g_{1}\right)$ and $\left(f, g_{2}\right)$ agree, and that if $g_{1}=g_{2} h$ for a radial homeomorphism $h: B^{n} \rightarrow B^{n}$, then $\epsilon\left(f, g_{1}\right)=\epsilon\left(f, g_{2}\right) h$. Thus $\mu\left(f, g_{1}\right)=\mu\left(f, g_{2}\right) h$, so that $\mu\left(f, g_{1}\right)$ and $\mu\left(f, g_{2}\right)$ are radially equivalent, and hence $\nu$ is well-defined.

Finally we check continuity. By noting that all of the subspaces of Euclidean space considered are compact, we observe that the topologies on the mapping spaces agree with the uniform metric topologies. It is then clear that all of the functions $\alpha, \cdots, \mu$ are continuous. Continuity of $\nu$ then follows by consideration of the following commutative diagram:

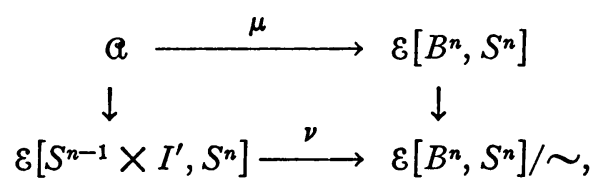

in which the left vertical map is the projection on the first factor and the right vertical map is the natural identification. Continuity of $\nu$ is an immediate consequence of the fact that the two vertical maps are identifications. 


\section{BIBLIOGRAPHY}

1. M. Brown, $A$ proof of the generalized Schoenflies theorem, Bull. Amer. Math. Soc. 66 (1960), 74-76. MR 22 \#8470b.

2. W. Huebsch and M. Morse, The dependence of the Schoenflies extension on an accessory parameter (the topological case), Proc. Nat. Acad. Sci. U.S.A. 50 (1963), 10361037. MR 32 \#439.

3. J. M. Kister, Microboundles are fibre bundles, Ann. of Math. (2) 80 (1964), 190199. MR 31 \#5216.

University of Auckland, Auckland, New Zealand 